\title{
Numerical investigation of pulverized coal aero mixture combustion at the presence of flow swirling
}

\author{
V.A. Kuznetsov ${ }^{\mathrm{a}}$, K.L. Morozov, and M.Yu. Chernetskii, Ph.D.
}

Siberian Federal University, Krasnoyarsk, Russia

\begin{abstract}
Numerical investigation results of burning pulverized coal aero mixture in the presence of swirl flow have been presented. The mathematical model has been chosen allowing describing correctly the pulverized coal combustion processes in the furnace with a swirl burner.
\end{abstract}

\section{Introduction}

According to the "Energy Strategy of Russia for the period up to 2030", approved by the RF Government No 1234-r of 28.08.2003, the solid fossil fuel will be the main source for energy production (in large power units) for the long term, therefore, the problem of increasing its use efficiency and economical expenditure is relevant. The coal power growth will be covered mainly through input units with traditional coal flaring.

Conducting experimental burns can not reveal fully the existing combustion process dependencies on the fuel quality and the physical and chemical laws of pulverized particles combustion processes. Consequently, a large role in the coal fuel burning technologies development and improvement is given to numerical modeling.

Mathematical modeling of combustion devices is today one of the most important ways to obtain the most representative information about aerodynamics, the local and overall heat exchange. Despite the great advances made in numerical experiment development, lots of variety, not fully researched coal structure and complex chemical processes occurring during coal fuel combustion, do not allow to create universal models. Therefore, it remains the task of finding relevant mathematical models using the existing experimental data on the coals and creating on their basis of an integrated model for the calculation of the combustion chamber, which will more accurately describe the combustion process in the combustion of pulverized coal-burning devices.

\footnotetext{
${ }^{a}$ Corresponding author: victor_partner@mail.ru
}

This is an Open Access article distributed under the terms of the Creative Commons Attribution License 4.0, which permits unrestricted use, distribution, and reproduction in any medium, provided the original work is properly cited. 


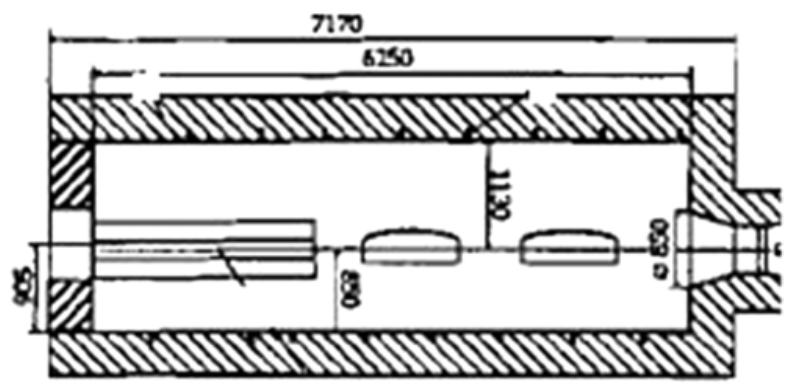

Figure 1. A combustion chamber.

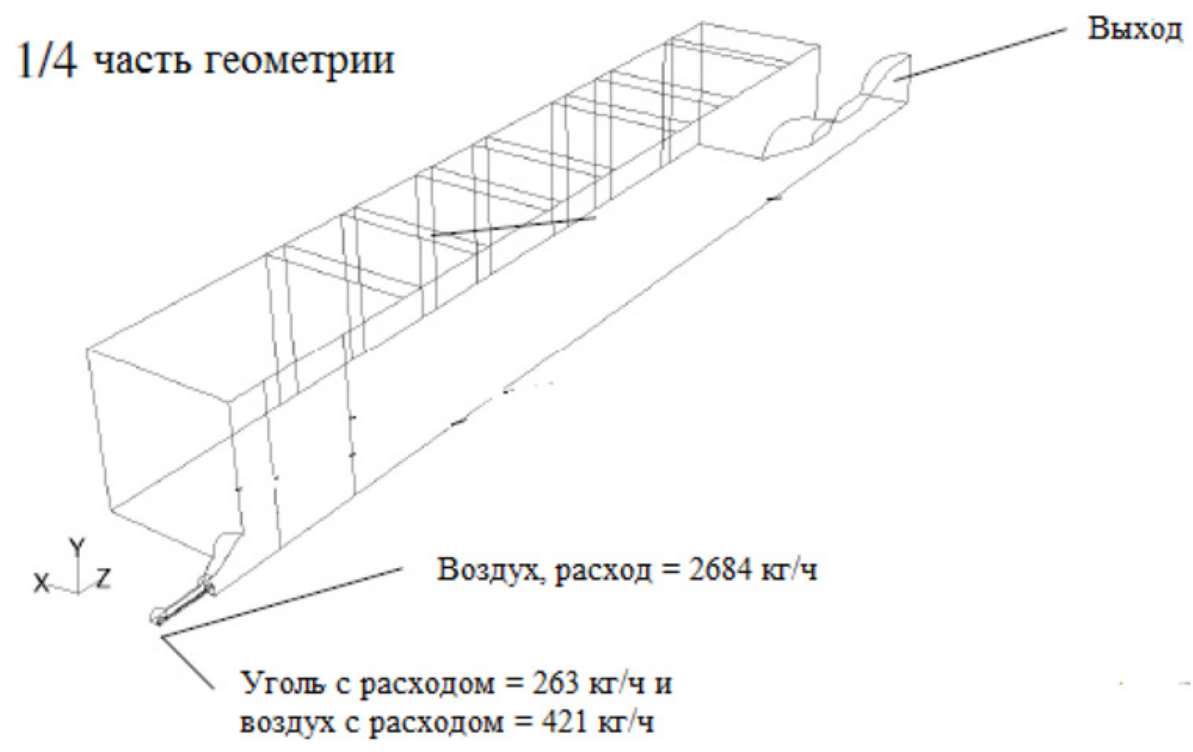

Figure 2. The problem geometry.

\section{Results and discussion}

Numerical investigation of pulverized coal aero mixture burning in the presence of flow swirl has been carried out using FLUENT CFD-package ver. 14 [2]. The computational research object has been chosen - a combustion chamber with swirl burner [1]. The furnace shape and dimensions are shown in Fig. 1.

For the calculations it was chosen a mathematical model, included: a carrier phase motion description, based on the RANS approach with a two-parameter turbulence model $k-\varepsilon$, radiation transfer based on the discrete-ordinate method, particles motion based on the Lagrange approach, combustion in the gas phase based on a hybrid model, coal particle burning included residual moisture output, devolatilization and coke residue combustion.

The task has an axial symmetry, so it has been used the 1/40f geometry. Figure 2 illustrates the problem geometry.

As the boundary conditions in the inner annular section input, the coal consumption has been specified of $0.0731 \mathrm{~kg} / \mathrm{s}$ and air flow with axial velocity of $23.02 \mathrm{~m} / \mathrm{s}$. In the outer annular section the air has been specified with parameters: the axial velocity of $43.83 \mathrm{~m} / \mathrm{s}$, the tangential velocity of $49.42 \mathrm{~m} / \mathrm{s}$. 
Thermophysical Basis of Energy Technologies

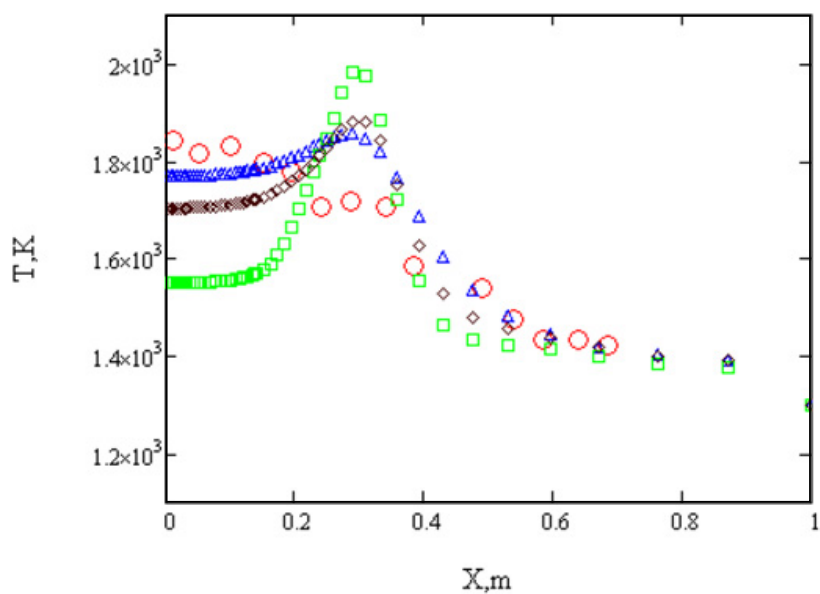

эксперимент

$\Delta \Delta \Delta \mathrm{k}$-е модель

वप् k-w модель

$\diamond \diamond \diamond$ sst модель

Figure 3. The temperature profile $(z=0.85)$.

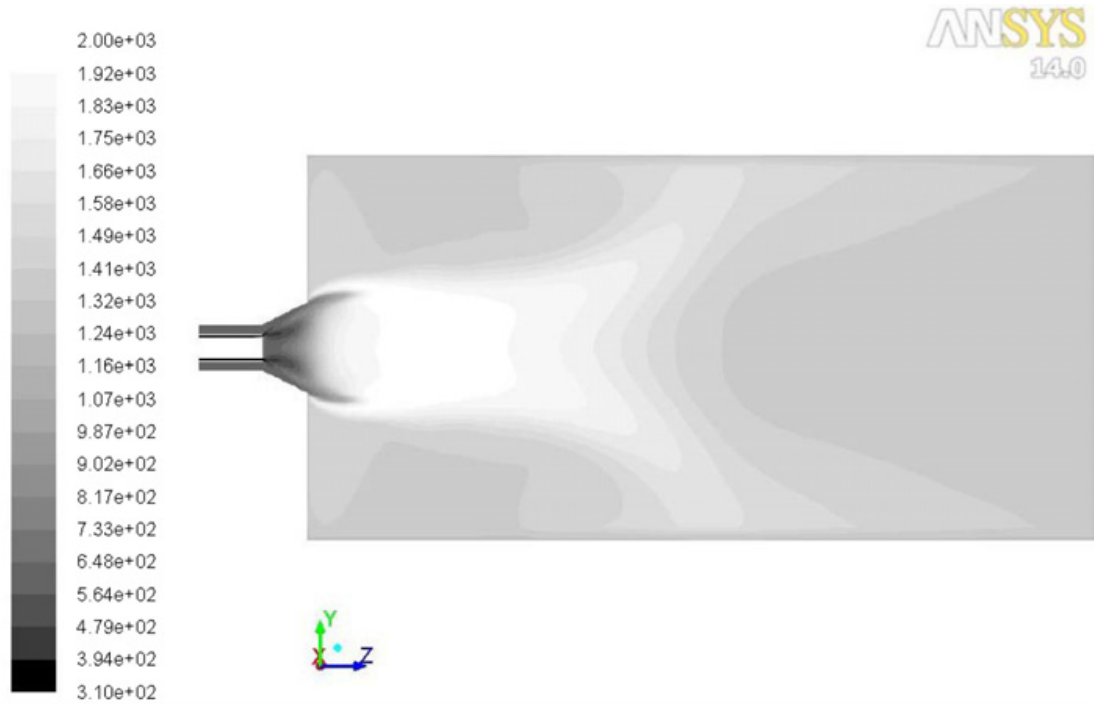

Figure 4. The temperature field.

The calculation results are presented down below. Different turbulence models comparisons are shown in Fig. 3. It is seen that the temperature profile, finding using the models $k-e$, is as close to the experimental temperature distribution. And using $k-w$ and sst it turns out, that the temperature is overestimated in areas where it should not be. The turbulence model $k-e$ is preferred for a given task. 


\section{Conclusion}

The calculations results and comparison with experimental data showed that the selected mathematical model and numerical methods for its solution allow describing correctly the pulverized coal combustion process in the furnace with a swirl burner and gives the opportunity to conduct numerical investigations of flaring solid fuel burning process with accuracy sufficient for engineering problems.

\section{References}

[1] André A.F. Peters, Roman Weber, Mathematical Modeling of a 2.4 MW Swirling Pulverized Coal Flame, Combustion Science and Technology (1997)

[2] I.K. Mitrofanov, FLUENT and GAMBIT - Programs for solving fluid and gas mechanics, heat and mass transfer tasks, Mode access http://www.teplota.org.ua/2009-08-25fluent_gambit_ru.html (2009) 